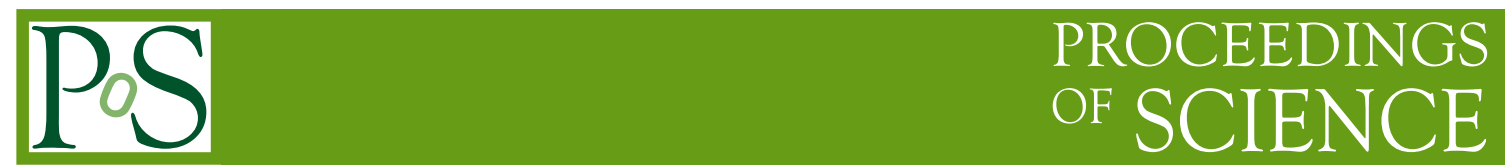

\title{
JEM-X: Six Years in Space
}

\section{Budtz-Jørgensen*, N.Lund, N.J. Westergaard, S.Brandt, J. Chenevez, C. A. Oxborrow and I. Lundgaard Rasmussen}

National Space Institute, Technical University of Denmark, Copenhagen, Denmark

E-mail: carlespace.dtu.dk

We report on the technical and scientific performance of JEM-X, the X-ray monitor on ESA's INTEGRAL mission. INTEGRAL has now been in orbit for more than six years, and the mission is foreseen to be extended beyond 2010. Overall, JEM-X performs very well, and can be expected to continue to do so for the duration of the mission. We discuss in some detail the operational experiences and the problems encountered with the microstrip detectors caused by the space environment and give a summary of the scientific results obtained. The analysis software is still being improved on, and we discuss briefly the significance of these improvements.

7th INTEGRAL Workshop

September 8-11 2008

Copenhagen, Denmark

* Speaker. 


\section{INTRODUCTION}

The role of JEM-X is to provide low energy coverage for the observations made in the hard $\mathrm{X}$-ray and gamma-ray bands by the two primary instruments on INTEGRAL: the Spectrometer (SPI) and the Imager (IBIS). JEM-X provides spectral and timing data in the 3-35 keV band and, simultaneously, more precise imaging than both SPI and IBIS.

\section{INSTRUMENT DESCRIPTION}

The X-ray detector system consists of two redundant imaging Micro-Strip Gas Chambers that view the sky through coded aperture masks. The specifications for one unit are given in Table 1. Only the most essential characteristics will be presented here, further details can be found in Budtz-Jørgensen et al. (2003) [1] and Lund et al. (2003) [2].

\subsection{The Coded Mask}

Each of the two instrument units has its own coded mask. The mask pattern is a hexagonal uniformly redundant array of the type described by Finger and Prince (1985) [3]. The mask elements are hexagons with a center-to-center separation of $3.3 \mathrm{~mm}$. The mask/detector separation $(3400 \mathrm{~mm})$ and the mask element size, determine the instrument angular resolution of $3.35 \mathrm{arcmin}$.

Only $25 \%$ of the mask elements are open. This small value for the open fraction was chosen in order not to overload the telemetry channel. As discussed by Gunson and Polychronopoulous, (1976) [4] and in't Zand et al., (1994) [5], a low value of the open fraction does not impact the limiting sensitivity of the instrument.

Table 1: JEM-X specifications (One Unit)

\begin{tabular}{|c|c|}
\hline Mask diameter & $535 \mathrm{~mm}$ \\
\hline Detector diameter & $250 \mathrm{~mm}$ \\
\hline Mask-detector distance & $3400 \mathrm{~mm}$ \\
\hline Energy-range & $3-35 \mathrm{keV}$ \\
\hline Energy resolution & $\Delta \mathrm{E} / \mathrm{E}=0.40(\mathrm{E}[\mathrm{keV}])^{-1 / 2}$ \\
\hline Angular resolution & $3 \operatorname{arcmin}$ \\
\hline Field of view (diameter) & $\begin{array}{l}4.8^{\circ} \text { Fully illuminated } \\
7.5^{\circ} \text { Half response } \\
13.2^{\circ} \text { Zero response }\end{array}$ \\
\hline Point source location & $30 \operatorname{arcsec}($ for a $10 \sigma$ source) \\
\hline $\begin{array}{l}\text { Narrow line detection sensitivity } \\
\text { Isolated on-axis source } \\
\text { For a } 3 \sigma \text { detection in a } 10^{5} \mathrm{~s} \text { observation }\end{array}$ & $\begin{array}{l}1.610^{-4} \text { phot } \mathrm{cm}^{-2} \mathrm{~s}^{-1} @ 6 \mathrm{keV} \\
1.310^{-4} \text { phot } \mathrm{cm}^{-2} \mathrm{~s}^{-1} @ 30 \mathrm{keV}\end{array}$ \\
\hline $\begin{array}{l}\text { Continuum sensitivity }(\Delta \mathrm{E}=0.5 \mathrm{E}) \\
\text { Isolated on-axis source } \\
\text { For a } 3 \sigma \text { detection in a } 10^{5} \mathrm{~s} \text { observation }\end{array}$ & $\begin{array}{l}1.210^{-4} \text { phot } \mathrm{cm}^{-2} \mathrm{~s}^{-1} @ 6 \mathrm{keV} \\
1.010^{-4} \text { phot } \mathrm{cm}^{-2} \mathrm{~s}^{-1} @ 30 \mathrm{keV}\end{array}$ \\
\hline Time resolution & $122 \mu \mathrm{s}$ \\
\hline
\end{tabular}




\subsection{The Detector Unit}

The detector is a Microstrip Gas Chamber with a nominal sensitive area of $500 \mathrm{~cm}^{2}$. The entrance window is $250 \mu \mathrm{m}$ Beryllium. The filling gas is a (90\%/10\%) mixture of Xenon and Methane at a pressure of 1.5 bar. The depth of the absorbing gas layer is $55 \mathrm{~mm}$.

A honeycomb collimator limits the detector field of view. The internal dimension of each collimator cell is $6.6 \times 6.6 \mathrm{~mm}$ and the height is $57.0 \mathrm{~mm}$.

The microstrip plate has orthogonal strip patterns on the front and back sides. The primary microstrip pattern has a pitch of $1062 \mu \mathrm{m}$ with $25510 \mu \mathrm{m}$ wide anode strips alternating with 458 wide cathode strips. The pattern on the back side has $1 \mathrm{~mm}$ wide strips with a $2 \mathrm{~mm}$ pitch. The readout electronics allows 2D X-ray position determination to better than $1 \mathrm{~mm}$ precision.

\subsection{On-Board Event Selection}

The count rates of the detectors are dominated by events caused by cosmic ray proton and alpha particles. Particle events are about 50 times as numerous as the X-ray induced events. The on-board rejection of the particle background is very efficient $(<99.5 \%)$, which is essential, since otherwise the telemetry channel would be overloaded by particle data.

\section{IN-FLIGHT TECHNICAL ISSUES}

During the first 6 years in space, both the hardware and the software of the instruments have generally performed according to expectations. However, the microstrip detectors themselves have posed problems which, although mostly solved, are complicating the instrument operation and data analysis, Budtz-Jørgensen et al. (2006) [6].

\subsection{Microstrip erosion}

Shortly after the activation of the detectors in space it became apparent that the microstrip anodes eroded at an alarming rate of one anode strip per day. It is believed that heavy cosmic ray ions can occasionally initiate electrical breakdowns close to the anode strips, see Budtz-Jørgensen et al. (2003) [1] and Lund et al. (2003) [2]. This situation forced changes in the planned operation of the detectors. The gas gain was reduced from the initial value of 1500 to 500 for both detectors and it was decided to operate JEM-X with only one detector (presently JEM-X1) activated at a time. Fortunately, the gain reduction diminished the damage rate to an acceptable level. After 6 years in space we can conclude that the loss rate is $\sim 4$ anode strips per year, and therefore, the survival of the instruments should be assured beyond 2010. The reduced gain has affected the performance to some degree, mainly because of the resulting decrease of the detector signals compared to the electronic noise.

\subsection{Gain Drifts}

The instruments have shown an unexpected ageing effect, namely a gradual increase in gain as a function of time of use (see Fig. 1). The effect is on the order of $0.7 \%$ of the original gain per orbit of use (3 days), corresponding to a $100 \%$ increase over a period of 1 year of active duty. 
The operational high voltage has by now been reduced more than 10 times in order to maintain the detector gain within a limited $\pm 10 \%$ range .

The gain change may be related to the diffusion of ions through the glass substrate whereby the electrical properties of the glass are changed, see for example Cicognani et al., (1995) [7]. (With more modern glass types it may be possible to avoid this type of gain drift).

The ageing effects of the glass substrate also include an increase in gain dependence on temperature variations. Initially, the gain increased $1 \%$ per degree. This has now increased to almost $4 \%$ per degree for the unit which has been operated for 5 years. However, the thermal environment is usually quite stable, and gain variations over an orbit are usually within $\pm 10 \%$.

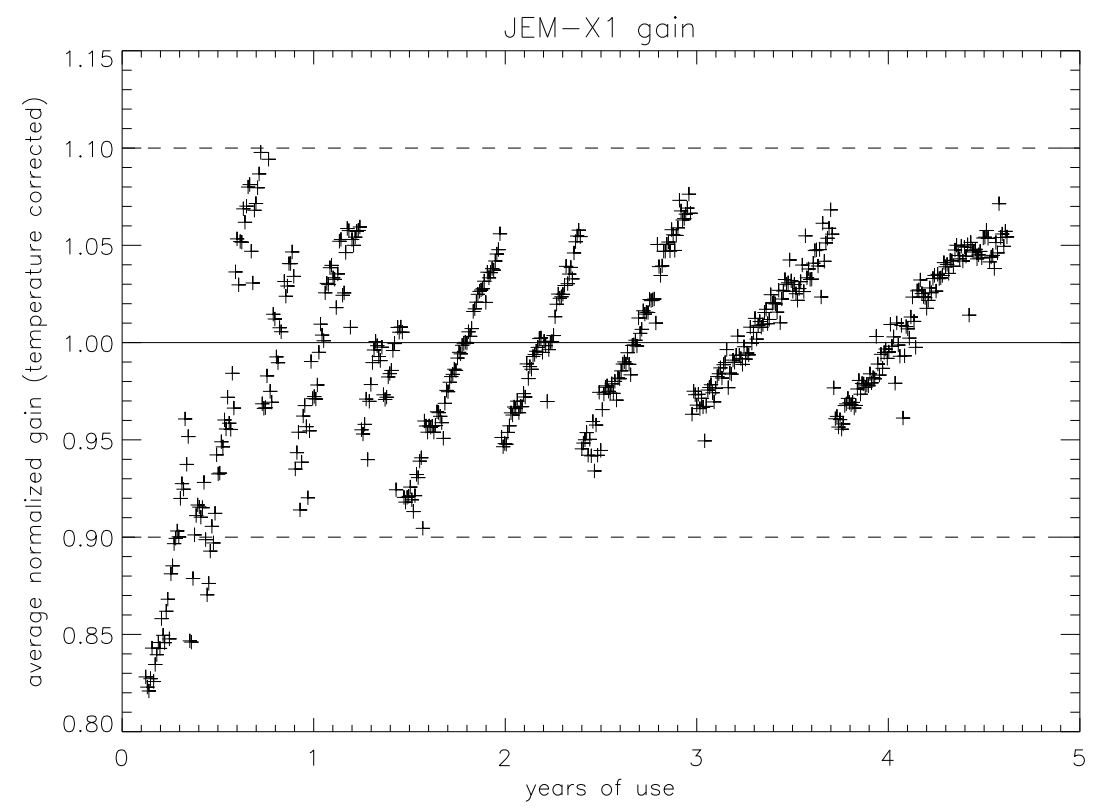

Figure 1: The gain evolution of the JEM-X1 unit as a function of time of use with the high voltage on. The data shows the average actual gain for each orbit. The gain is kept within a $\pm 15 \%$ band by adjusting the high voltage setting at appropriate intervals.

\subsection{Electronic Efficiency}

The reduction of the gas gain has resulted in a modified low energy response of the detectors. The efficiency of the electronics is decreased due to a poorer signal to noise ratio. The efficiency at the electronic threshold is affected, but the particle rejection is also less efficient and results in a loss of true X-ray events. The detector efficiency is determined by two terms. One term, the quantum efficiency, is dependent only on the energy of the incoming photon and can be accurately calculated from absorption in the Be detector window and the interaction with the gas volume. The second term, the electronic efficiency, is dependent only on the pulse heights of the signals generated by the electron avalanche process. This term is therefore dependent on the actual gas gain. We have determined the electronic efficiencies of the two detectors in space by observing the Crab on axis with different settings of the detector gain. We could then in a self-consistent manner derive the electronic efficiency, see Budtz-Jørgensen et al. (2006) [6]. 


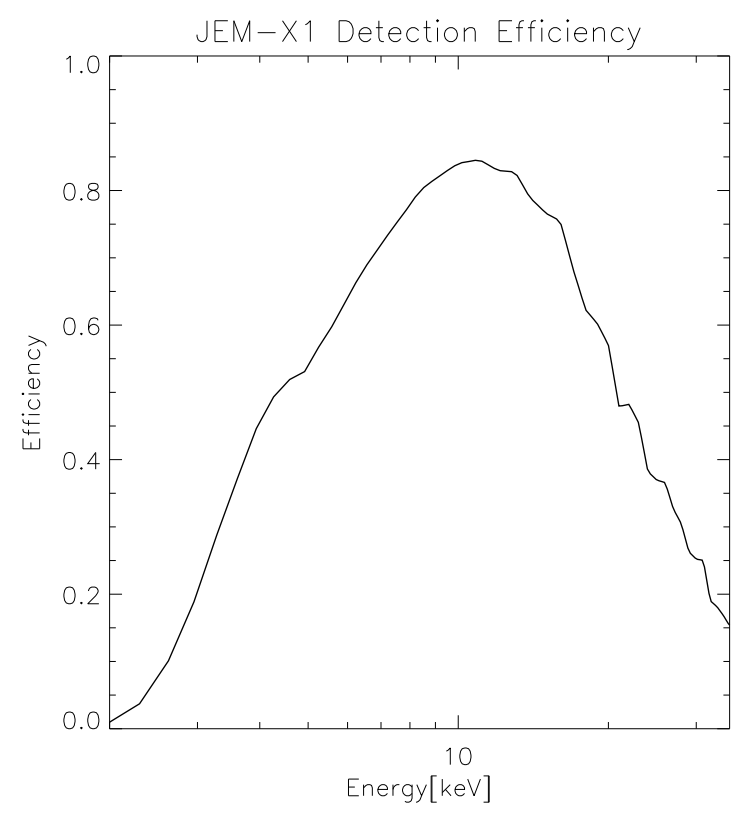

Figure 2: The JEM-X1 on-axis efficiency in the 2 - $35 \mathrm{keV}$ band, at the nominal gas gain of 500 .

\section{IN-FLIGHT PERFORMANCE}

\subsection{Spectroscopic Performance}

The contribution of the electronic noise to the detector energy resolution is insignificant, even at the reduced gain of 500, compared to the statistical contribution from the avalanche processes of the detectors. Therefore the gain reduction in itself did not degrade the energy resolution of the instruments.

The detectors have after 1 year of pre-launch testing and 6 years of operation in space shown no signs of degradation due to contamination of the detector gas. This has been verified after launch on several occasions.

The evolution of the energy resolution is derived from the detector response to the four calibration sources and from the Xenon background line. The energy resolution at $22 \mathrm{keV}$ has, over the mission, only degraded from about $9.5 \%$ to about $10.5 \%$. The gain evolution discussed above is, as may be expected, slightly non-uniform across the micro-strip plate. Therefore the spatial gain map, needed to convert pulse height to energy, needs to be updated at about 1 year intervals. The in-flight spatial gain map is derived by using the Xe fluorescence lines of the background.

\subsection{Imaging and Source Positioning}

The INTEGRAL pointing accuracy is better than a few arcseconds and therefore, in most cases does not contribute to the source localization error. The alignment of the instruments with respect to the INTEGRAL star trackers was determined using selected point sources with well known positions. The sample consisted of more than 3500 detections, covering a time interval of more than one year. The Crab Nebula was not used in this connection because it is diffuse and the position of the centroid of the emission is energy dependent. 
The instrument alignment analysis employed specially developed IDL routines generating images from which the source positions were extracted using 2D Gauss fitting.

This analysis, Budtz-Jørgensen et al. (2006) [6] made it clear that the alignment of the telescopes correlate with the mask temperature ( 0.4 arcsecond/degree). The mask temperature, which is strongly correlated with the INTEGRAL Sun aspect angle, varies between $-40{ }^{\circ} \mathrm{C}$ and $0{ }^{\circ} \mathrm{C}$. The observed temperature dependence therefore implies that the telescope alignment can vary by up to 17 arcseconds with respect to the star trackers.

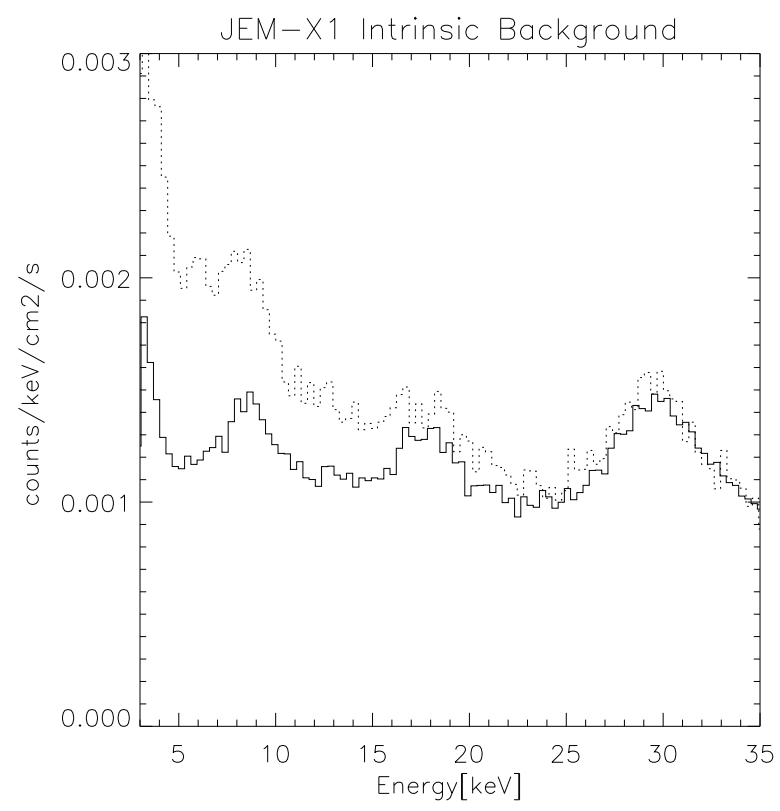

Figure 3: The solid curve displays the intrinsic background of JEM-X1 derived from the INTEGRAL Earth occultation campaign. The dotted curve displays the total detector background found for an extragalactic empty field observation.

\subsection{Detector Background}

The background has been derived from empty field observations. When INTEGRAL is outside the Earth's radiation belts the background rate after particle rejection is about $20 \mathrm{cts} / \mathrm{s}$ in the 4 to 35 $\mathrm{keV}$ range Special background conditions existed during the 4 Earth occultation observations which were undertaken by INTEGRAL in February 2006. During this campaign the JEM-X Field of View was fully covered by the Earth for a sufficient time (in total $\sim 3$ hours) to allow determination of the intrinsic detector background.

The solid curve in Fig. 3 displays the intrinsic background of JEM-X1, while the dotted curve displays the total detector background typically found for empty field observations. Correction for Earthshine contributions has not been attempted. The spectral peaks seen in Fig. 3 stem from $\mathrm{K}$-fluorescence in the collimator $(\mathrm{Cu}, \mathrm{Zn}$ and $\mathrm{Mo})$ and from $\mathrm{K}$-fluorescence in the Xe detector gas.

The background intensity across the detector has a noticeable increase towards the edge of the detector. This indicates that a large fraction of the background comes from photons generated in material around the detectors. 
There has been no indication of a significant long term increase in the intrinsic background caused by radiation activation of the detectors or their surroundings. We have, however, since the launch of INTEGRAL seen a significant increase $(100 \%)$ of the total trigger rate as demonstrated in Fig. 4. The total trigger rate is dominated by the flux of cosmic ray particles passing through the detectors. At present this flux is unusually high due to the deep Solar minimum. The trigger rate shown in Fig. 4 correlates very well with cosmic particle rate measured by ground observatories such as the Oulu cosmic neutron detection system. It is remarkable that the residual background rate, after onboard particle rejection, is almost unchanged since launch. this confirms the high efficiency the particle rejection technique employed.

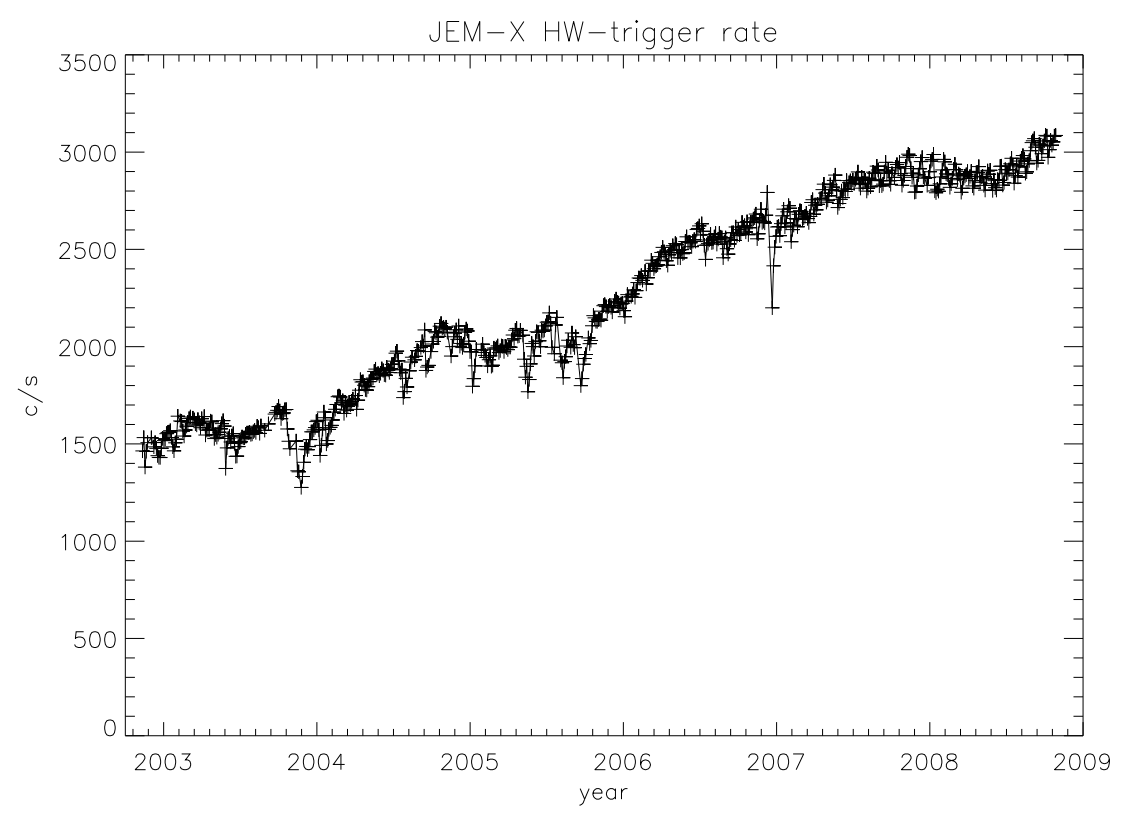

Figure 4: The JEM-X1 Hardware trigger rate as a function of mission time.

\subsection{Event Timing}

X-ray events detected by JEM-X are time-tagged onboard with a resolution of $122 \mu$ s. During ground calibration JEM-X timing was calibrated relative to the INTEGRAL onboard clock, and the phase stability of the $122 \mu \mathrm{s}$ time bins was verified to within a few $\mu$ s.

In orbit, the absolute timing (meaning timing relative to UTC) of the full chain of JEM-X instrument, spacecraft, and ground segment was verified by using observations of the Crab pulsar. See Brandt et al. (2003) [8]. The absolute phase of the $33 \mathrm{~ms}$ Crab pulsar is regularly monitored and accurately determined by radio observations. These observations verified the absolute timing stability of the full system to be within $\pm 100 \mu \mathrm{s}$.

\section{JEM-X DATA ANALYSIS}

The data handling follows the general scheme set up by the ISDC, Oxborrow et al. (2006) [9]. The gain changes with time during a revolution. The energy tagging is done by monitoring this 
change with the built-in calibration sources. The quality of this process is monitored by determining the position of the Xe fluorescence peak in the instrument background spectrum.

The slow deterioration of the anodes is also monitored as well and Instrument Characteristics (IC) files with calibration data are produced for a number of epochs throughout the mission and distributed by ISDC.

The standard data analysis is controlled by a script in the Offline Science Analysis package (OSA, currently version 7) that calls the executables from the Instrument Specific Software. It extends to the imaging and extraction of fluxes, spectra, and lightcurves for individual sources. The end-point in the standard analysis is the merging of sky-images from several science windows into mosaics, and a search for weak sources not seen in individual images.

The Instrument Specific Software is still being developed. The next release will feature improved corrections for the variations in the electronic efficiency seen over time. This will make it possible to compare with greater precision observations made with different JEM-X units at different times.

For more details see Westergaard et al. (2003) [10].

\subsection{Source Detections}

The image reconstruction in OSA consists of a back-projection technique whereby each count is projected to the (multiple) positions in the sky where it could have originated considering the obstructions imposed by the mask, the collimator, or other structural elements. The image is subjected to a "flatfielding" process whereby large scale structures are subtracted, improving the visibility of the peaks from celestial sources.

These peaks are identified and compared with the image variance. The minimum acceptance criterion is that the source must be seen in more than one energy interval, the source search is done in three standard energy bins.

\subsection{Source Detection Limits}

The source detection sensitivity for single science windows was evaluated based on about 5000 source detections. Fig. 5 displays the source detection limit as a function of off-axis angle for an exposure time of $2000 \mathrm{~s}$. The sensitivity was determined as the lower limit of the intensity of known sources that have been found in a blind search in all public data upto November 2007. The dashed lines give comparison levels for the Crab Nebula. The figure refers to observations with only a single unit .

\subsection{Analysis Using Mosaics}

The source detection sensitivity can be significantly improved by merging several individual images using the OSA mosaicking tool developed at DTU Space. This tool combines sky images pixel by pixel, each pixel weighted by its variance value, to produce mosaic intensity and variance maps. A detailed description of the tool is available in Chenevez et al. (2004) [11]. The mosaicking of mosaics is also possible, allowing the user for instance, to combine both JEM-X1 and JEM-X2 mosaic images of the same sky region.

The mosaic sky images can be read by the ISDC tool "mosaic_spec" to derive the flux directly from the image at some specified coordinates, thus making it possible to build a source spectrum 


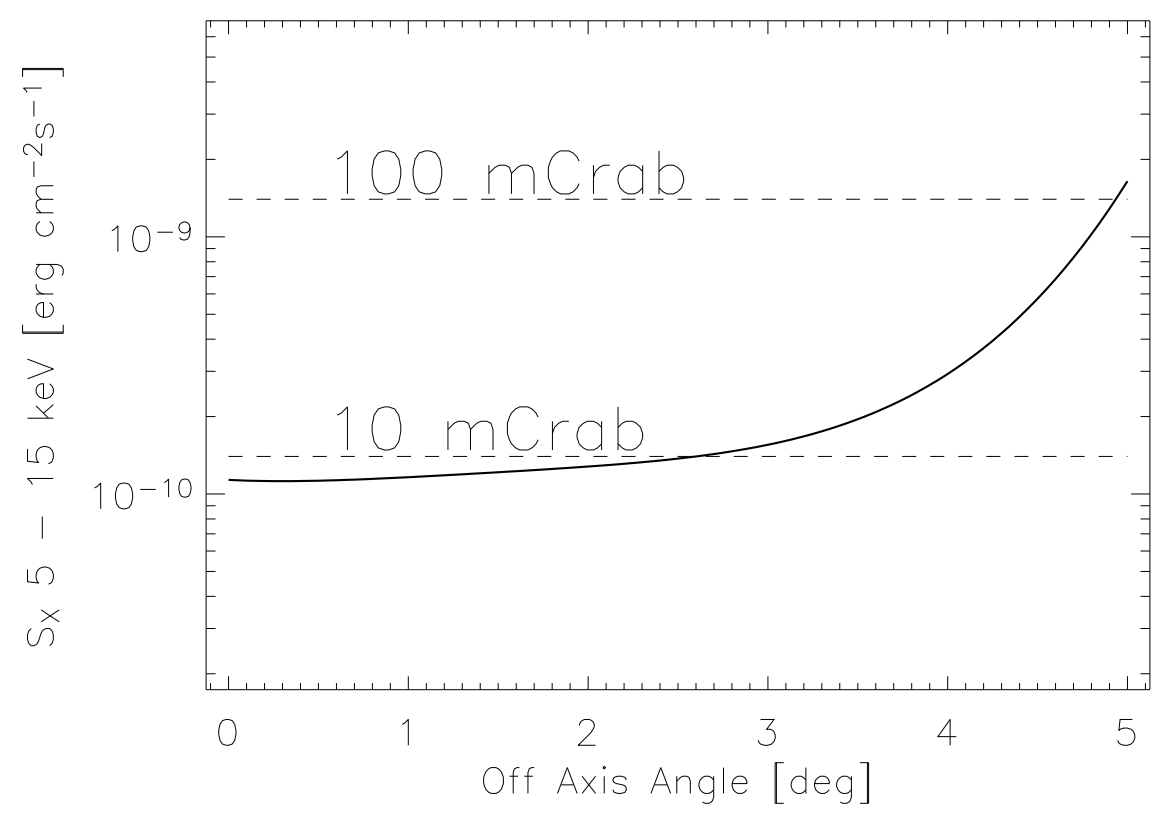

Figure 5: The JEM-X source detection limit as a function of off-axis angle for an exposure time of 2000 s. The sensitivity has been determined as the lower limit of the intensity of known sources that have been found in a blind search of all public data upto November 2007. The dashed lines give comparison levels for the Crab Nebula.

based on the user defined energy bands. This method can be used to obtain the spectrum of weak sources not detected in individual pointing images. Spectra obtained by this method are also less prone to cross talk between nearby sources compared to the OSA component j_lc_spectra. (See the Known Issues in the JEM-X Analysis Cookbook at ISDC).

\section{SELECTED SCIENTIFIC RESULTS}

\subsection{JEM-X Source Catalogue}

A source catalogue for the first three years of operation has been published in Westergaard et al. (2007) [12]. Since then, the sky coverage has increased as illustrated in Fig. 7 although, the emphasis is still on the central regions of the galaxy.

An updated catalogue is being constructed based on all available public data (i.e. up to revolution 605). So far, 145 sources have been identified in the images. Of these, there are 48 HMXBs, $70 \mathrm{LMXBs}, 2 \mathrm{CVs}$, and 7 other binaries of unknown nature. Of other galactic sources 4 SNRs and 1 solitary star have been found. The extragalactic sources seen are: 1 cluster of galaxies and 10 AGN. Finally, there are 2 new sources of unknown classification. The ISDC general reference catalog version 29 has been used for this summary (see web address:isdc.unige.ch/Data/cat/latest).

\subsection{Source Catalog Derived From Mosaic Images}

A total of 140 X-ray sources have been detected so far by means of the JEM-X mosaic images. For instance, the first detection of the source SWIFT J174535.5-290135.6, as well as the discovery 


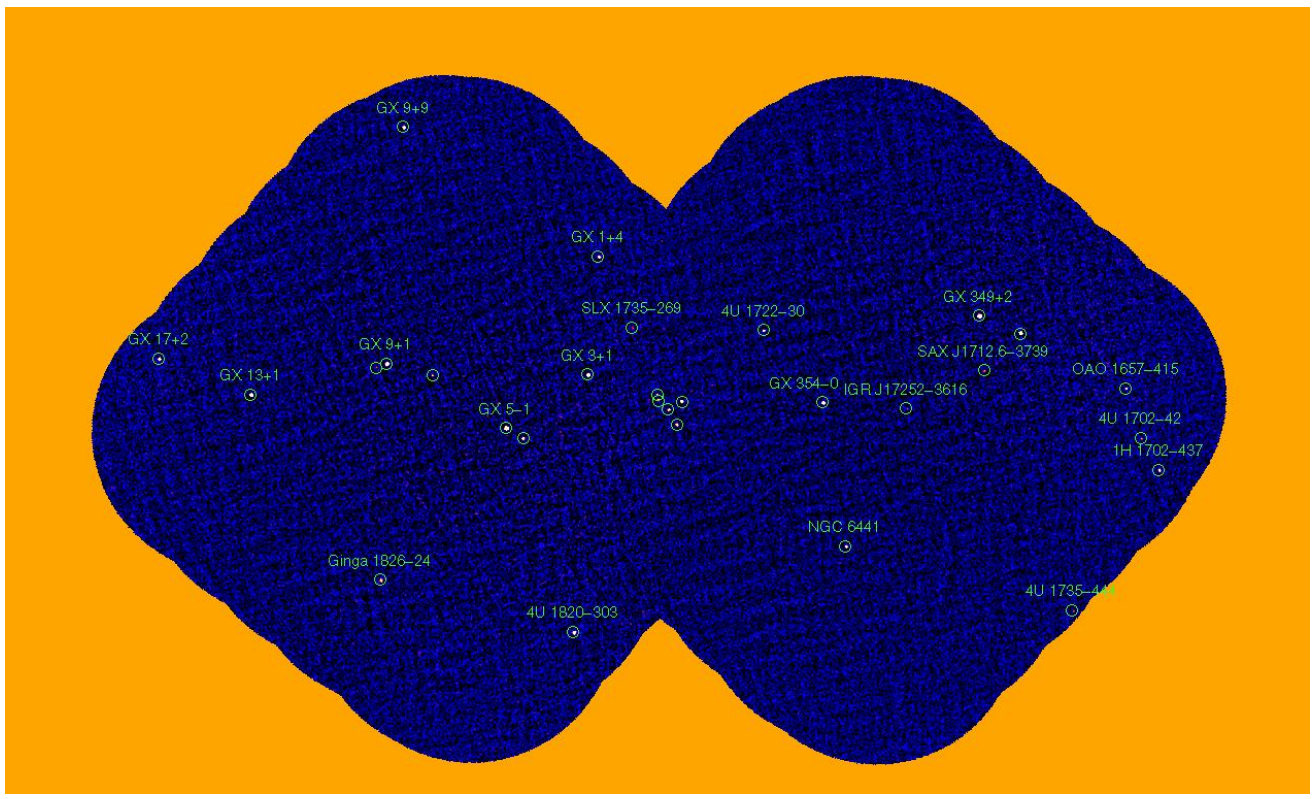

Figure 6: Mosaic of 350 individual pointings around the Galactic Centre region taken during the AO-4 Key Programme between February 26th and March 25th, 2007 (INTEGRAL revolutions 534 to 542). The image shows the significance map in the $5-25 \mathrm{keV}$ band, and a total of 30 sources can be seen with a significance > 5 .

of the new source IGR J17454-2703 have been achieved in this way. (Chenevez et al., 2006) [13] (Kuulkers et al., 2007) [14]

The deepest effective exposure in the Galactic Centre now exceeds 3 Ms for JEM-X, allowing a detection limit of $1 \mathrm{mCrab}$ between $3-10 \mathrm{keV}$ and $0.3 \mathrm{mCrab}$ between $10-25 \mathrm{keV}$ (see the JEM-X Observer's Manualfor plots of the source detection limit as a function of observation time).

The overlap between the standard catalog and the catalog of sources found in the mosaic images is 93 sources. The deeper search for mosaic sources thus results in 47 sources not found in the standard search based on single science window detections. The mosaics have so far only been made for a limited part of the JEM-X sky coverage and that is the reason for the 53 sources with no mosaic counter part.

The total number of sources spotted by JEM-X is therefore 192 .

\subsection{X-Ray Burst Studies}

Since the beginning of the INTEGRAL mission six years ago, the JEM-X team at the DTU Space has been monitoring the X-ray burst activity recorded by the satellite. Thanks to the wide field of view of its instruments and its long sky monitoring, INTEGRAL is a mission very well suited to study the high energy emission of a large number of X-ray bursts, especially for rare and peculiar events. A number of observations have shown that X-ray burst data are indeed easily analyzable with JEM-X (3-35 keV) and IBIS (18 keV - $10 \mathrm{MeV})$. For instance, half of the rare intermediate long bursts known to date have been observed by INTEGRAL (e.g., Chenevez et al.(2008) [15]). A convenient data method to study X-ray bursts is to start by making a JEM-X detector light curve (a standard OSA product), from which it is possible to register times of the 


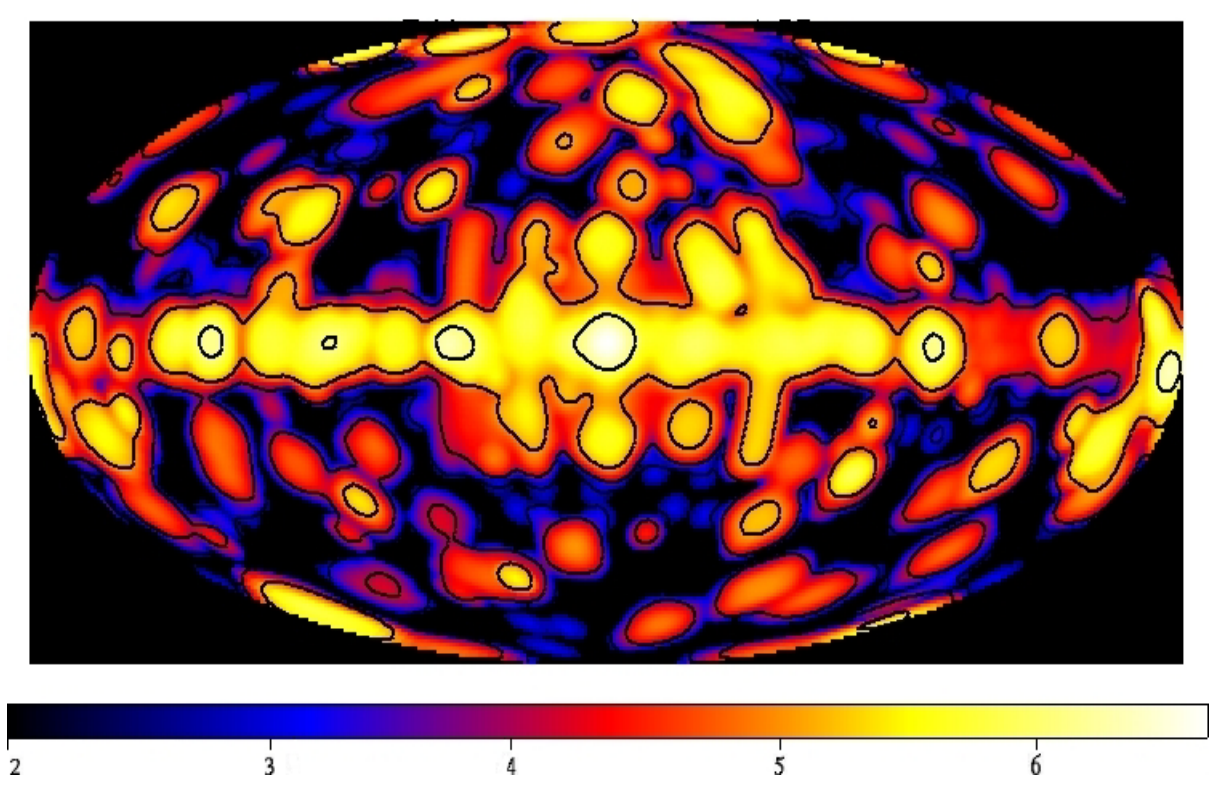

Figure 7: The map shows the total exposure time (vignetting included) over the entire INTEGRAL mission in a logarithmic color scale. The contours are shown for integer exponents to 10 (also given in the color scale) of exposure time in seconds. In the center of the galaxy the maximum is $4.7 \times 10^{6} \mathrm{~s} .16 \%$ of the sky is not covered by JEM-X at all but $50 \%$ has more than $10^{4} \mathrm{~s}$ of effective observation time as estimated with the peaked through-put as a function of off-axis angle.

bursts happening in the FOV of the instrument. To identify the sources of the bursts it may be necessary to compare short exposure images around the times of the bursts with reference images of the same observation, but outside the burst period. Then source extracted light curves and spectra, corrected for vignetting, can be used to analyze the burst emission. A systematic check of X-ray bursts from archive data indicates that more than 100 bursts per year have been detected using JEM-X alone. Currently, a database of more than 1000 bursts has been created from archive data.

\section{Conclusions}

The operability of JEM-X has been verified, and the spectral and spatial resolutions have been found to be consistent with the values calculated prior to launch. The source localization capabilities actually significantly exceed the specification values.

The larger than expected sensitivity of the microstrip detectors to the space environment (presumably induced by heavy cosmic rays) has threatened to limit the lifetime of the instruments. This has forced us to reduce the operating voltage and therefore the gas gain of the microstrip detectors. Having taken this precaution we are convinced that JEM-X will stay operational beyond 2010.

The in-orbit performance was reviewed and considerable improvement in the understanding of the instrument performance in the space environment has been achieved. 


\section{References}

[1] C. Budtz-Jørgensen, N. Lund, and N. Westergaard et al., Proceedings of SPIE 5165, pp. 139-150, 2003.

[2] N. Lund, C. Budtz-Jørgensen, N. Westergaard, and S. Brandt et al., A \& A 411, pp. L231-L238, 2003.

[3] M. H. Finger and T. A. Prince in Proceedings of the 9th Intenational Cosmic Ray Conference, OG-9, p. $295,1985$.

[4] J. Gunson and B. Polychronopoulous MNRAS 177, p. 485, 1976.

[5] J. J. M. in't Zand, J. Heise, and R. Jager $A$ \& A 288, p. 665, 1994.

[6] C. Budtz-Jørgensen, N. Lund, N. J. Westergaard, S. Brandt, C. A. Oxborrow, J. Chenevez, I. L. Rasmussen, S. Laursen, S. M. Pedersen, J. Polny, P. Kretschmar, O. Vilhu, M. Feroci, F. Frontera, G. Juchnikowski, V. Reglero, S. Martínez-Núnez, S. Larsson, A. Zdziarski, and S. Fahmy in Proceedings of SPIE, 6255, pp. 62662z-1, 2006.

[7] G. Cicognani, P. Convert, A. Oed, and J. Pannetier in International Workshop on Microstrip Gas Chambers, p. 235, 1995.

[8] S. Brandt, C. Budtz-Jørgensen, and N. Lund et al., A \& A 411, pp. L433-436, 2003.

[9] C. Oxborrow, J. Chenevez, P. Kretschmar, S. Larsson, S. Maisala, and N. Westergaard, “Instrument Specific Software for JEM-X: Architectural Design Document," in Documentation pages of ISDC, Version 10.0, 2006.

[10] N. J. Westergaard, N. Lund, C. Budtz-Jørgensen, and S. Brandt A \& A 411, p. L257, 2003.

[11] J. Chenevez, N. Lund, N. J. Westergaard, C. Budtz-Jørgensen, P. Kretschmar, and R. Walter in Proceedings of the 5th INTEGRAL Workshop, The INTEGRAL Universe, ESA-SP 552, p. 837, 2004.

[12] N. Westergaard, J. Chenevez, N. Lund, C. Budtz-Jørgensen, and S. Brandt in Proceedings of the 6th INTEGRAL Workshop, The Obscured Universe, ESA-SP 622, p. 275, 2006.

[13] J. Chenevez, C. Sanchez-Fernandez, and E. Kuulkers et al., ATEL 756, 2006.

[14] E. Kuulkers, S. Shaw, and A. Paizis et al., A \& A 466, p. 595, 2007.

[15] J. Chenevez, M. Falanga, E. Kuulkers, S. Brandt, N. Lund, and A. Cumming in Proceedings of the 7th INTEGRAL Workshop, An INTEGRAL View of Compact Objects,'PoS(Integral08)033ì 2009. 